\title{
Evolution of IS Competencies due to Mandatory Telework on Organizational scale - A Work-Systems Approach
}

\author{
Harshit Kumar Singh \\ Indian Institute of Management Ahmedabad \\ phd16harshits@iima.ac.in
}

\begin{abstract}
Given the recent pandemic crisis, telework has become a mandatory practice for organizations across the world. Mandatory large scale implementations of telework are an abrupt technological shift in organizations, which presents a unique set of challenges for organizational competencies. In this research initiative, we derive from the work systems framework to analyze the consequences of telework shift on organizational competencies from a resourcebased view. Many organizations are inexperienced and unprepared to implement telework on an organizational scale. We analyze how organizations have been affected by these large scale implementations of mandatory telework and how the competencies of these organizations have been affected. Content and thematic analysis of several interviews of managers across organizations supervising teleworking teams have been done to provide insights into how IS competencies have been affected by this shift.
\end{abstract}

\section{Introduction}

Telework, also known as telecommuting or workfrom-home, is the phenomenon of working from home using technology, and not physically commuting to the work location (Nilles, 1991). Although telework has been a field of research for a few decades, the recent pandemic threat of COVID-19 forced organizations worldwide to implement mandatory telecommuting for most of their employees on an organizational scale (Frenkel, 2020). This implementation creates a sudden technological shift in organizations which warrants organizational changes.

Organizations that are well-managed on the business front are often constrained by their capacity to evolve with technological shifts. Gaining advantage through strategic investments into areas such as business opportunities, customer, and market analysis is short-term if these practices are dependent on existing technologies (Rothaermel, 2016). Technological and managerial changes are necessary if these organizations are to sustain gained advantages.

\author{
Sanjay Verma \\ Indian Institute of Management Ahmedabad \\ sverma@iima.ac.in
}

Rigid structures and processes can cause these wellmanaged firms to lose advantage if technological change is missing in the face of technological shifts.

We explore the effect of telework as a technological shift on Information Systems (IS) competencies and present the analysis of the impact. We borrow from the resource-based view of organizations and use organizational competencies as the focus of our analysis. Organizational competencies have been defined as unique or superior capabilities that organizations develop over time and are difficult to replicate. We show a prominent shift towards the evolution of IS competencies due to this technological shift.

Three unique aspects differentiate this research initiative from the studies done in the extant literature on telework. First, we focus on the 'mandatory' aspect of the telework in this study, which was a mute concept in previous studies. There is a plethora of research done periodically on understanding the reason for the non-adoption of telework on a large scale (Atkin and Lau, 2007; Martin and MacDonnell, 2012; Silva, 2019). However, our current context is different, as the notion of choice-based adoption becomes non-existent due to the mandatory nature of telework in the context of the pandemic outbreak. Second, the context in this study is different from all the earlier studies as the scale of telework is much larger than those focused in the literature. Telework has mostly been practiced on a small scale as a complete shift to telework has never been seen as a reasonable or practical scenario (Silva, 2019). Third, the shift to telework in organizations on this scale was abrupt. This difference presents an opportunity to explore telework as a technological discontinuity. Organizations were not prepared for this change, and hence the competencies were affected on an organizational scale that we explore in this paper.

Our research objective is to find out what are the levels of changes in IS capabilities of digital functions across organizations due to the technological discontinuity caused by the mandatory telework implementation on an organizational scale. To generate insights on this technological shift and its effect, we interviewed managers and telework experts from the industry who are currently leading teams over 
telework. In doing so, we aim to provide insights for organizations to follow best practices for telework implementations and present a framework to analyze future technological discontinuities. The boundary of our research was constrained to experts from organizations that work in digitally-driven or supported functional areas. Thematic analysis is used to code data collected to generate themes. We use the work system framework to analyze the themes and explore the effect of mandatory telework on organizations.

\section{Background and Literature Review}

\subsection{Telework}

Traditionally teleworking is seen as an aspiratory practice from an employee's perspective and has been focused for its positive impact on employees, such as increased job satisfaction (Chung and Van der Horst, 2018). Telework has been an active area of research for over two decades but was never adopted enthusiastically by organizations (Martin and MacDonnell, 2012; Silva, 2019). There are mixed results on the effect of teleworking on organizational outcomes. While most studies point to the positive effect of teleworking on organizational outcomes (Golden and Veiga, 2008; Martin and MacDonnell, 2012), some have focused on the adverse effects (de Vries et al., 2019).

However, the implementation of telework on a large scale comes with its challenges. This large scale and mandatory implementation of telework is a technological and managerial shift on several fronts in an organization. Many organizations are inexperienced in managing large scale telecommuting as it was previously considered difficult to adopt (Silva, 2019). They are unprepared to manage the organizational challenges that emerged from this abrupt shift.

We adopt a resource-based view to explore the effect of telework on organizational competencies. Earlier studies on telework that employed a resourcebased view provide evidence that suggest telework affects organizational competencies (Illegems and Verbeke, 2004). These provide evidence for positive as well as negative effects of teleworking on different competencies. Further, there is evidence that adopters have a more positive outlook to telework as compared to non-adopters pointing to a bias against the practice.

\subsection{Technological discontinuity, Competencies, and IS research}

Tushman and Anderson (1986) categorized technological discontinuities as competency-enhancing or competency-destroying. A competency-destroying technological discontinuity is one where the existing expertise is rendered useless by the new technology, and a complete rework is required to rebuild the competency from scratch. On the other hand, a competency-enhancing technological discontinuity allows organizations to build on the existing competencies to evolve and improve the expertise that already exists in the organizations. We use this categorization to analyze the effect of telework on the IS competencies of the organizations. We also add a competency-sustaining category to classify those elements which are unaffected by the technological discontinuity as done in previous research of similar nature (Petrie, 2004).

IS competencies and capabilities have been researched in detail to establish the relation between firm performance and IS capabilities, as shown by Aydiner et al. (2019). Tangible and intangible assets, including hardware and software, human capital, employee skills, corporate culture, IS administrative skills, and experience have been classified as part of IS competencies. Research in IS have used similar categorizations to analyze IS capabilities such as managerial, analytical, and technical capabilities (Lin, 2020). Studies have explored IS competencies to provide evidence of the contribution of these competencies to gain competitive advantages in firms (Zhang and Lado, 2001).

\subsection{Work System Framework and IS Theory}

The importance of work system theory in IS discipline has been formalized and presented as a framework to understand IS-reliant systems in a series of articles by Alter (Alter, 2003; 2008; 2013). He argues that the work system framework provides a model for understanding different parts of an IS reliant system and can be used to generate a static and dynamic view of the system. It can be a useful tool to understand problem areas in the organization and explore opportunities to evolve. The work system consists of nine elements, with four primary elements representing the system itself - the processes and activities, participants, information, and technologies, and five other elements that supplement the understanding of the work systems. These are products/services produced, customers, environment, infrastructure, and strategies.

We derive from the literature of the work-system in IS research and use the framework as the guiding model of our analysis. This analytical approach to use work systems framework to analyze IS competencies is a novel approach. IS researchers have called for a systems perspective of IS-reliant organizations that go 
beyond treating systems as an artifact (Alter, 2013). We use the framework to understand how the competencies of different elements present in the organizations have been affected while treating the participants and processes as an integral part of the system and competencies. We can see a clear overlap between elements of the work system framework and IS competencies such as IS infrastructure, IS human resources, and IS administration (Aydiner et al., 2019). The framework provides us a model to analyze the competencies by breaking them down to different parts through the nine elements of the framework.

\section{Methods}

To maintain the robustness and rigor of our study, we collected data in two stages and used a hybrid of inductive and deductive coding mechanism (Fereday and Muir-Cochrane, 2006). The first corpus was inductively coded and used to develop a template for the deductive coding of the second corpus. The second corpus was then coded using a hybrid coding technique, which involves first deductively coding the data using the template generated and then revising and modifying the developed themes inductively.

For the first stage of our study, we emailed an open-ended survey to 94 managers across several industries. The email mentioned the study's objective, and an option for respondents to remain anonymous or provide their email ids for further discussion on the topic. The first part of the questionnaire asked the participants to list the challenges they encountered while working from home, and the second part asked them to list down the strategies used to mitigate or counter these challenges. We received responses from 32 managers, 24 of whom agreed to engage in further correspondence if needed, and 8 chose to remain anonymous. Participants' responses were coded and further categorized into broader themes using content analysis. The two principal investigators inductively coded the responses and developed initial themes that were then discussed and merged. Any coding conflicts, if present, were resolved through detailed discussions between the investigators.

For the second part of our study, we contacted a different set of 72 managers across different organizations for semi-structured interviews. The email mentioned the objective of the study and details on the areas of discussion. The questions for these interviews also included questions formed based on the results obtained from the first corpus. We received responses from 32 managers, and we interviewed 30 of them, who were working within digital economies. All the managers' interviewed were handling teleworking teams for at least three months at the time of the interview. The participants consisted of 23 males and 7 females. The age of 18 participants was in the range of $30-40$ and 12 in the range of 40-50. The number of people they interacted with via telework daily was in the range 5-10 for 2 participants, $10-20$ for 23 participants, and more than 20 for 5 participants. The functional areas that the participants worked in covered marketing, sales, support, research and development, information technology, business management, product management, human resources, and operations. Most of the participants were managing employees from multiple functional areas. The industries covered included software and IT, e-commerce, healthcare, education, banking, telecommunications, retail, FMCG, and media organizations. Online consents were collected for each interview through Google forms as in-person meeting was not possible due to the pandemic.

Participants were asked in detail about the effect of telework on various areas within the organizations and the differences from the non-teleworking scenario. They were then asked specifically about the effect of telework on competencies of the firm. The interviews were then transcribed. The transcripts were anonymized by removing participants' names, their organizations, or any other identifying information relating to the participants. After transcribing the interviews, the two principal investigators developed a coding scheme focused on the challenges that the telework presents to organizational capabilities. Initially, a deductive coding scheme was used, based on the themes that emerged from the content analysis of the first corpus. These themes were then revised and modified based on the inductive analysis of the data with open, axial, and selective coding sequentially. Both the investigator individually coded the transcripts and any coding conflict, if present, were resolved between the investigators similar to the first stage.

\section{Results}

From the first stage of our study, 11 major themes emerged from the coded responses for the challenges faced during teleworking. Table 1 presents the themes and the number of respondents who mentioned the particular challenge. For strategies to counter the challenges, 12 themes emerged, which have been listed in Table 2. These are the steps taken by teleworkers to mitigate the challenges that arise from telework.

For the second stage of our study, 22 axial themes (second-order themes) emerged with 12 themes on the challenges faced while teleworking and 10 themes on the strategies used to counter the challenges. The challenges gave insight into how a particular competency was affected, and their counter-strategies 
provided insight into what level were the existing systems modified to analyze the destructive, sustaining, or enhancing effect on the competency. The aggregate themes that emerged from the last stage of the coding process (selective coding) are the challenges emerging from telework and the strategies to counter challenges in telework. Figure 1 gives a brief of the data structure used for obtaining the themes, as suggested by Gioia et al. (2013). There were overlaps with the themes that had emerged in the first part of our study, and we also saw new themes emerging from the interviews. Next, we discuss each of the themes explaining how telework affects different aspects of the organizational competencies.

\begin{tabular}{|c|c|}
\hline \multicolumn{2}{|c|}{ Table 1: Challenges emerging from telework } \\
\hline Theme & Frequency \\
\hline Lack of resources & 27 \\
\hline Work/Home role conflict & 24 \\
\hline Psychological distress & 17 \\
\hline Time issues & 14 \\
\hline Work organization and management & 13 \\
\hline Interdependent work & 11 \\
\hline Lack of face-to-face communication & 10 \\
\hline Managerial, Supervisory issues & 10 \\
\hline Organizational distrust & 10 \\
\hline Technological limitations & 5 \\
\hline Security concerns & 3 \\
\hline
\end{tabular}

\begin{tabular}{|c|c|}
\hline \multicolumn{2}{|c|}{ Table 2: Strategies to counter the challenges } \\
\hline Theme & Frequency \\
\hline Routine reinforcements & 10 \\
\hline Communication with coworkers & 8 \\
\hline Change in physical environment & 8 \\
\hline Define virtual communication rules & 7 \\
\hline Leveraging improved technology & 7 \\
\hline Modified processes and tools & 7 \\
\hline Planning ahead & 6 \\
\hline Restructuring and alignment of goals & 4 \\
\hline Communication with family & 4 \\
\hline Morale reinforcement & 4 \\
\hline Increase flexibility & 4 \\
\hline Task organization & 3 \\
\hline
\end{tabular}

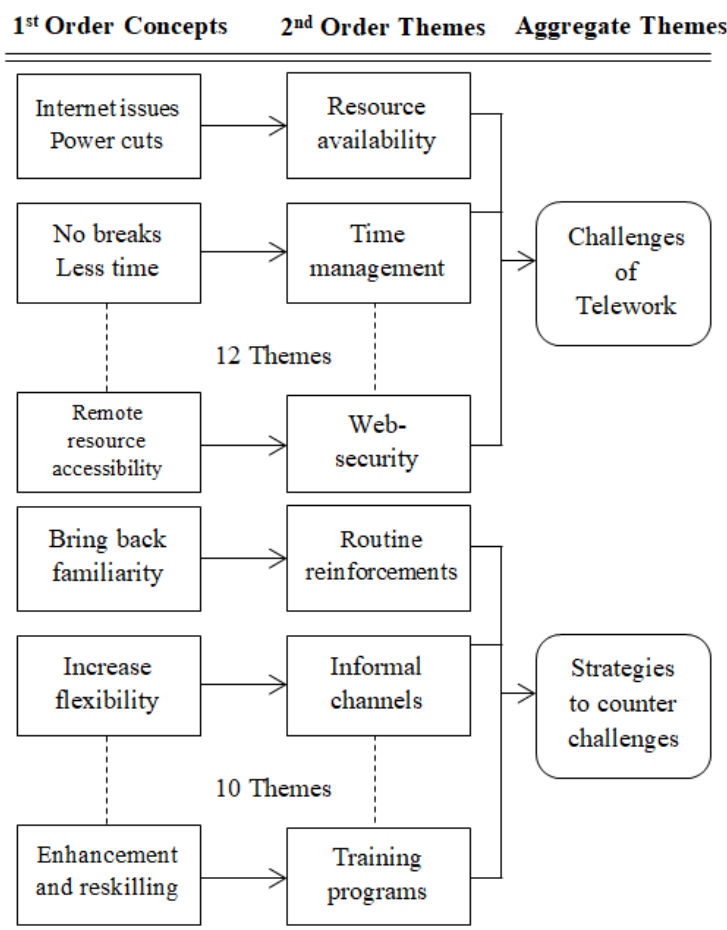

Figure 1: Data Structure

\subsection{Themes in challenges emerging from telework}

4.1.1. Resource Availability. One of the most frequent challenges listed was a lack of resources. Office spaces have dedicated resources that are used to assist work tasks for employees. Many employees not only find this assistance convenient but also necessary. Working without these becomes a challenging assignment as the casual home resources are not sufficient to replace the dedicated office resources. The most common lack of resources listed was a lack of appropriate internet connection. Many employees do not have a high-speed internet connection in their homes as in the pretelework phase, its usage was limited. Further, with most companies mandating work from home, internet traffic has increased exponentially, causing the regular internet speed to go down even for those who have a high-speed internet connection. The other sub-themes of this challenge include problems with power-cuts, lack of soundproof rooms to attend calls/meetings, lack of proper table/chair (ergonomics), and lack of assistive resources like monitors and headphones.

4.1.2. Time Management. The time-related issue is one of the primary challenges of teleworking. Firstly, with teleworking, end of work time is not defined as employees are often expected to go beyond official work hours. Some participants mentioned the work 
hours extending till late into nights. Secondly, the mismanagement of time also affects the eating and sleeping cycles of employees and, in some cases, reduces the number of breaks received while working from home.

4.1.3. Role conflict. The family-work role conflict arises out of the psychological boundary overlap between the two roles. This interferes with work routines and causes a "lack of workplace environment", a term frequently emerging in the data gathered by this study. An important aspect of this mismatch is the domestic disturbance that comes as a part of this conflict. Managing family distractions and work efficiency becomes a challenge for the teleworker. The disruption in routine is reciprocal. As family distractions interfere with work routines, the work interferes with family routines as well. Finally, this challenge is amplified further if more than one person is teleworking in the same home, an increasingly common occurrence with large scale implementation of work from home.

4.1.4. Team Management. With the decrease in face to face communications, supervisorial and managerial challenges also emerge. Monitoring and guiding subordinates is difficult with telecommuting. It becomes difficult for supervisors to create sync among different teams. Accessing the productivity and accountability of delegated tasks also becomes more complicated as measuring outcome is not always an appropriate measuring criterion.

Completion of interdependent tasks is the next cited challenge on the list. While teleworking, many tasks that require collaboration and cooperation between individuals and teams become inefficient as the processes of collaboration and cooperation become challenging. This also extends to tasks such as brainstorming or ideation, where individuals work together to come up with solutions and ideas. Without physical access to other individuals, these processes become too complex to function efficiently.

4.1.5. Technical Limitations. The technological limitation is another challenge that emerged in our results. For instance, when the participants in a meeting are substantial in number, communication over calls on the internet becomes impossible. There are no alternatives to conferences, which are an annual practice by large organizations. Many managers express the need for additional features in the emeeting software to make meetings more productive, like a feature that mutes all other participants when one of the participants is speaking. Another aspect of this is the lack of technological knowledge by members of organizations. Training sessions have to be scheduled in order to learn new tools implemented for teleworking, and often traditional businesses find it challenging to engage with customers over telecommunication channels due to a lack of technical know-how.

4.1.6. Lack of face to face communication. Lack of face to face communication is another major challenge that affects work efficiency while teleworking. Workplace strategies such as negotiation or persuasion in processes like sales are not as effective when carried over calls or emails. Further, the inability to share physical items such as physical models or print-outs increases the complexity of processes. Businesses dependent on customer involvement are affected as client involvement becomes completely virtual. A lack of direct interaction limits the use of physical cues, which often form an essential part of business routines.

4.1.7. Work Management. Disorganization of work is another issue commonly faced by the teleworkers. Participants reported an uneven workload across days and a decrease in work speed leading to decreased productivity. More time is spent on organizing work via calls, which results in slower execution of the work. Prioritization of work becomes an additional task, and unscheduled calls affect efficiency.

Participants cite the inexperience in virtually managing all work-related tasks as the root of this challenge. The workload gets unevenly distributed across teams and team-members, often leading to overwork for some employees. Some participants also reported having more work than they have on regular days, while others were relatively free. As employees save time in commuting, few organizations expect them to utilize this time for office work, and few others introduce more training programs as they expect employees to be relatively free.

4.1.8. Increased competition. Another phenomenon that emerged from telework was an increase in competition among employees after shifting to telework. Employees lack direct contact with managers and superiors that earlier observed their productivity and efficiency in-person. To overcome this lack of direct visibility, the environment in organizations has become more competitive as employees are motivated to prove their contribution through the work completed.

4.1.9. Business Tactics. Business tactics have been affected by telework on different fronts. First, as mentioned earlier, strategies such as negotiation, persuasion, which required face-to-face interactions, 
have been hampered. Second, the use of physical cues to read situations, which were very important to managers facing clients, has also been affected as this becomes difficult over virtual channels. Third, engaging clients to continue business associations with them has been affected by telework. These involved initiatives like informal meetings with clients and periodical client visits to increase their involvement and understanding in business activities.

4.1.10. Increased stress levels. An increase in psychological distress is among the most commonly reported challenge. Words such as "boring", "lost drive", "lack of concentration", "frustration", "emotional trauma", and "stress" have been used to describe the psychological challenges faced during mandatory telework. This challenge is an important one to analyze and understand as it highlights one of the key differences from the traditional teleworking challenges. Traditionally, working from home was a personal choice, and often positive or mildly negative psychological outcomes were associated with it like loneliness and worry (Mann and Holdsworth, 2003). As work from home is implemented on a large scale and mandated, we can see new and more aggressive psychological challenges emerging.

4.1.11. Organizational distrust. An interesting challenge that emerged out of the collected data was an increase in organizational distrust. As face-to-face communication decreases, misunderstandings are more frequent and lead to the dissipation of trust (Nandhakumar and Baskerville, 2006). Managers become distrustful of employees as they are unable to confirm when an employee is deceiving them, for instance, by lying about internet connectivity problems. More status updates and calls are expected by superiors, and support from top decreases due to lack of transparency while teleworking.

4.1.12. Web security. Another challenge mentioned is related to security challenges over confidential data accessibility and secure access to proprietary technology. Often accesses to these technologies are location-based, which becomes impossible to acquire with teleworking.

\subsection{Themes on Strategies to counter challenges in telework}

4.2.1. Routine reinforcements. In terms of strategy to counter the challenges, the most common strategy that emerged is reinforcing routines. Adhering to timelines and discipline is an essential part of completing work in most businesses. The vigilance requirement in terms of following routines requires extra effort than before. Reinforcing routines via reminders and alarms helps in regaining control and successfully follow timelines.

4.2.2. Communication with coworkers. More frequent and structured communication with colleagues is the next most frequent theme that emerged. As discussed earlier, a lack of face-to-face communication often causes miscommunication and unclear exchange of instructions among team members. Establishing a more concrete communication process, such as writing detailed minutes of meetings, reconfirming action items, or sending the requirements in advance helped in diminishing those effects. Even if physical presence is not possible, virtual face-to-face communication provides psychological presence (Panteli and Duncan, 2004).

4.2.3. Change in physical environment. Changing the home environment to better support the teleworking process is the next strategy commonly used by teleworkers. These include improving home equipment, allocating separate space for work at home, and buying additional furniture to support working conditions. These changes aid the psychological transformation to a work-related mindset at home while teleworking.

4.2.4. Define virtual communication rules. Defining new rules and guidelines for virtual meetings is the next strategy for countering the challenges faced while telecommuting. These rules add structure to the phenomena of telecommuting and improve the efficiency of meetings. Some examples of these rules are muting by all non-speakers, raising hands before speaking, ensuring only one speaker speaks at a time, greeting coworkers via chat messages. The guidelines mentioned are expected to become norms and promoted as telecommuting manners to be standardized over time. Similar results have been reported in the literature for successful communication in virtual teams (Curşeu, Schalk, and Wessel, 2008).

4.2.5. Leveraging improved technology. Leveraging advanced technology helped in countering many challenges across organizations. For instance, when facing challenges while communicating via standard office software, employees switched to other means such as messaging apps and social media platforms. Some suggested the automation of follow-up procedures to reduce the time consumed in the process. Having back-up alternatives ready in the event of technology failure is another successful strategy. These technologies are formalized over time and integrated with existing tools and processes. 
4.2.6. Training programs. One of the most common themes was an increase in training programs in organizations. This was aimed at not only the employees but also towards customers to better equip them with the knowledge needed to leverage the evolved and modified technological changes in tools, processes, and software. These training programs were necessary for organizations to improve the capabilities of the stakeholders involved in the organization.

4.2.7. Modified tools and processes. In addition to using alternative technologies, participants also mentioned the use of new or modified processes and tools to oppose the adverse effects of telework. As the methodology of work changes, the artifacts involved in the process are also expected to evolve to facilitate the change. For instance, the technology usage patterns are monitored by some organizations to aid the evaluation of employee's performance and productivity.

4.2.8. Informal channels. Another reoccurring theme was an increase in the push from the organization for informal interactions between the employees. This was done to overcome the lack of affiliation and relatedness that the employees feel due to the physical separation from the organization. Further, these channels became a way of managing and reducing employee stress levels. Additionally, these now act as forums to take feedback to improve the processes and activities inside the organizations and to measure the success of already modified processes and activities.

4.2.9. Planning ahead, Restructuring and alignment of goals. Advanced planning was an intuitive solution that emerged out of the collected data. For instance, planning calls in low network congestion time helped reduce the problems faced due to low bandwidth. The foresight involved in planning tasks is significant and requires analysis of patterns. Another important step was the restructuring of goals. These can be achieved by following steps like redefining KPIs and revising deliverables by team members.

4.2.10. Communication with family and increased flexibility. Participants mentioned the importance of discussing the challenges faced with family members as an effective way to mitigate the challenges. The family is now an immediate part of the working environment and needs to be in alignment with the teleworker. Others mentioned the importance of the need for more flexibility in work attitude to meet the changing environment. An improved organization of workload, while identifying and eliminating unnecessary calls was also listed by a few respondents.

\section{Discussion}

In this section, we discuss in detail each aspect of the work system framework and how the IS competencies were perceived to be affected by the technological shift. We will also discuss those aspects of work-systems that were not affected by the technological discontinuity for exhaustive analysis. We categorize them based on the extent of the redesign that each of the elements underwent. The categories are named as competency-enhancing, competencysustaining, and competency-destroying (adapted from Tushman and Anderson, 1986).

\subsection{Customers}

Competencies relating to the management of customers are among the primary competencies that have been affected by this technological discontinuity. While the customers themselves remain mostly unaffected, the lack of face-to-face interaction that forms one of the biggest challenges of telework directly affects the processes and activities relating to customers, which are discussed in detail in the later sections.

The only effect that can be seen on customers directly is the lack of technical knowledge of customers in handling new tools and software that are introduced as part of evolving IS competencies. This effect can be classified as a competency-enhancing discontinuity as training programs and tutorials are being developed by organizations to equip customers with knowledge to handle the technological shift. This competency-enhancing process is visible in the data we collected and the themes that emerged from the data.

\subsection{Products and services}

Products and services are the least affected area within the boundary of our analysis. As we have analyzed IS driven or supported functionalities in our study, the nature of existing products and services remains unchanged.

Organizations may plan to introduce new services that leverage the technological shift towards teleworks in time. However, as the period in which we do our study is still in the early stages of the technological shift, we did not come across any organization that planned to develop new products or services leveraging telework.

Based on the evidence gathered, we conclude that the competencies related to products and services have remained largely unaffected, and hence we classify them under the competency-sustained category. 


\subsection{Processes and activities}

The competencies relating to processes and activities are most adversely affected by this technological discontinuity. Existing processes and activities proved inadequate to handle the shift to telework. As the nature of the communication changes to virtual for geographically co-located teams, many of the existing processes and activities were unusable and are forced to be redesigned. We classify competencies relating to processes and activities as competencydestroying.

Multiple processes have been affected due to telework. We discuss a few of the processes here, but the list is in no way exhaustive. First, the processes in organizations such as the evaluation of employees heavily rely upon managers' feedback on employees' work efficiency. Managers did this evaluation not only via output measure but also by continuous monitoring of work tasks in-person daily. With telework, the managers are either forced to evaluate employees through only output or existing reporting systems are evolved and redesigned. These reporting systems include more monitoring tools and processes as compared to earlier systems and are different.

Second, processes involving task-management have been reworked in organizations, as seen in the results of our analysis. The existing process mostly involved 10-15 minutes every day chat before starting work and were often informal. As most teams worked in geographically same locations, task redistribution was a common phenomenon within teams in organizations as in-person communications were synchronous. These processes are now more formalized, and there is an added overhead of communication time if any task is redistributed as meetings have to be scheduled, and all stakeholders involved have to be made available. This is different as compared to earlier, as the process has become asynchronous due to the change in the nature of communication.

Third, processes involving updates are similarly affected. Updates on completion of small tasks add overhead to efficiency in a similar manner, as explained in processes involving task-management. These processes are made more structured in nature with new tools in place to synchronize communications. The use of the group feature of the text message application 'WhatsApp' was one of the most commonly reported changes.

Similarly, activities in the organizations which were part of organizational competencies were affected due to telework. One such example of the competencydestroying effect is on customer relations. Activities such as 'client-meets' to hosting organizations are no longer possible. Organizations are forced to redesign their customer relations strategies and start new activities such as virtual catch-ups with clients.

\subsection{Participants}

Participants are the employees of the organization. Telework has also affected the attributes and attitudes that are required for employees to function efficiently in an organization. Our research shows that managers have begun to focus on employees with higher selfefficacy and who are self-motivated. These are required characteristics in post-telework adoption, as many organizations are moving towards output measuring evaluation schemes, as mentioned earlier. With this shift, monitoring and push from managers to complete tasks will reduce, and self-motivated employees are expected to be more efficient. Thus, we classify the effect of telework on competencies related to participants as enhancing. Organizations will evolve their workforce over time to include these attributes, which have become necessary due to this technological shift.

Additionally, few organizations have introduced new roles that are tasked with managing telework related communication and coordination activities. These roles further add to the capabilities of the organizations to leverage the technological shift, hence enhancing participant-related competencies.

\subsection{Information}

Information flowing inside the organizations has been unaffected by the shift towards telework as the nature of information, codified or non-codified, remains the same. As discussed earlier, although the process and tool involved in the flow of information have been affected, the competencies relating to the information itself are sustained.

\subsection{Technologies}

As technology is an integral part of telework implementations (Golden, 2009), technological competencies of the organizations have been enhanced due to telework. Although existing technologies have been retained, many new tools and software have been introduced in organizations to ensure productivity and efficiency improvements. These include new communication tools, updated security technologies to enable work from home, new coordination and remotetracking tools, and other such technological updates. Technological competencies of organizations have been enhanced with this shift. 


\subsection{Environment}

Telework has shaken the environmental capabilities of organizations on many levels. The shift from dedicated office spaces to virtual offices has completely changed the nature of the organizations' environment. The organizational culture, which involves norms and expected behaviors, has been challenged due to the change in the work environment's nature. A push to change organizational culture emerges in our analysis. The regulatory practices have been redesigned and modified, as discussed earlier. Employees become more competitive as with telework, the visibility of employees decreases in organizations (Illegems and Verbeke, 2004), which is also an emerging theme in our results. Thus, we classify environmental competencies as being destroyed by the technological shift.

\subsection{Infrastructure}

Infrastructural competencies, similarly, have been adversely affected by telework. Many organizations have previously invested substantially in infrastructure, but a shift to work from home renders all the infrastructures useless. Organizations are forced to reanalyze the effectiveness of existing infrastructures with instances where major firms are planning the reduction of physical office spaces it occupies.

Additionally, firms are forced to shift the dedicated equipment and technological infrastructure to employee's homes, a common theme in our data. These include infrastructural support from basic office furniture to specialized hardware such as IP phones and multiple screens. Internet stability and capabilities are also enhanced by providing additional financial support to employees for these facilities. Hence, we classify infrastructural competencies as destroyed from the technological discontinuity.

\subsection{Strategies}

For strategies, we found that the effect on competencies depends mostly on the nature of the business as it heavily drives the strategies. We found mixed results in our data, with a few organizations mentioning strategical competencies being destroyed and others reporting strategies mostly unaffected. The organizations that have been affected by telework are mostly dependent on a face to face communication for business.

Table 3 summarizes the effect of telework as a technological discontinuity on the competency area we discussed in this section, which has been derived from the work system framework.

\begin{tabular}{|c|c|}
\hline $\begin{array}{c}\text { Table 3: Effect of telework as a technological } \\
\text { discontinuity on the competency areas derived } \\
\text { from the work system framework }\end{array}$ \\
\hline $\begin{array}{c}\text { Competency Areas } \\
\text { Customers }\end{array}$ & Competency-enhancing \\
\hline Products/services & Competency-sustaining \\
\hline Processes and activities & Competency-destroying \\
\hline Participants & Competency-enhancing \\
\hline Information & Competency-sustaining \\
\hline Technologies & Competency-enhancing \\
\hline Environment & Competency-destroying \\
\hline Infrastructure & Competency-destroying \\
\hline Strategies & \\
\hline & \\
\hline
\end{tabular}

\section{Limitations and Future Directions}

Here we discuss a few of the limitations that were present in our analysis. First, although one of the greatest strengths of this initiative was the in-depth and detailed analysis of individual cases in several organizations, this was limited by gender diversity, which is reflective of the lack of diversity in the industry. Second, we interviewed only the managers of the companies and analyzed the effect on different areas of competencies from their perspective. A casebased analysis will further shed light on the nature and extent of the effect of telework on IS competencies. Third, as this is an early stage of the technological shift, the effect and evolution of competencies are expected to continue over the next few months. Similar periodic studies might provide insight into any new aspects of telework that are not yet visible.

\section{Implications and Conclusion}

Insights from our paper provide a framework for the effective enhancement of IS competencies for organizations while shifting to telework. It provides a list of focus area which needs change in organizations. Further, the insights from our paper, in general, have implications for understanding and improving organizational competencies in the aftermath of an unexpected technological shift. We provide evidence of the necessity of organizational change when faced with a technological shift. Lastly, we also show how the work system framework can be used to measure the extent of the effect of a technological shift or discontinuity on an organization. The process followed 
in this study can be used as a framework to analyze, understand, and counter future discontinuities.

To conclude, we explored telework as a technological discontinuity due to recent events that forced organizations to implement telework on a large scale. Our analysis shows how organizations have evolved or redeveloped existing competencies and capabilities in the face of technological discontinuities. We also presented a framework to analyze organizational competencies from a work-systems perspective combined with the resource-based view. Our results show an overall evolution of competencies relating to customers, employees, and technologies. A redesign of competencies in processes and activities, environment, and infrastructure is needed as the existing competencies are destroyed in these areas. The results have implications for planning and countering future technological discontinuities.

\section{References}

[1] Alter, S. (2003). 18 Reasons Why IT-Reliant Work Systems Should Replace" The IT Artifact" as the Core Subject Matter of the IS Field. Communications of the Association for Information Systems, 12(1), 23.

[2] Alter, S. (2008). Defining information systems as work systems: implications for the IS field. European Journal of Information Systems, 17(5), 448-469.

[3] Alter, S. (2013). Work system theory: overview of core concepts, extensions, and challenges for the future. Journal of the Association for Information Systems, 72.

[4] Atkin, D. J., \& Lau, T. Y. (2007). Information technology and organizational telework. Communication Technology and Social Change: Theory and Implications, 79.

[5] Aydiner, A. S., Tatoglu, E., Bayraktar, E., \& Zaim, S. (2019). Information system capabilities and firm performance: Opening the black box through decisionmaking performance and business-process performance. International Journal of Information Management, 47, 168-182.

[6] Chung, H., \& Van der Horst, M. (2018). Women's employment patterns after childbirth and the perceived access to and use of flexitime and teleworking. human relations, 71(1), 47-72.

[7] Curşeu, P. L., Schalk, R., \& Wessel, I. (2008). How do virtual teams process information? A literature review and implications for management. Journal of Managerial Psychology.

[8] de Vries, H., Tummers, L., \& Bekkers, V. (2019). The benefits of teleworking in the public sector: Reality or rhetoric? Review of Public Personnel Administration, 39(4), 570-593.

[9] Fereday, J., \& Muir-Cochrane, E. (2006). Demonstrating rigor using thematic analysis: A hybrid approach of inductive and deductive coding and theme development. International journal of qualitative methods, 5(1), 80-92.

[10] Frenkel, S. (2020, March 06). The Week in Tech: Welcome to the Age of Mandatory Videoconferencing. New York Times. Retrieved from https://www.nytimes.com/

[11] Gioia, D. A., Corley, K. G., \& Hamilton, A. L. (2013). Seeking qualitative rigor in inductive research: Notes on the Gioia methodology. Organizational research methods, 16(1), 15-31.

[12] Golden, T. D. (2009). Applying technology to work: Toward a better understanding of telework. Organization Management Journal,6(4), 241-250.

[13] Golden, T. D., \& Veiga, J. F. (2008). The impact of superior-subordinate relationships on the commitment, job satisfaction, and performance of virtual workers. The Leadership Quarterly, 19(1), 77-88.

[14] Illegems, V., \& Verbeke, A. (2004). Telework: what does it mean for management?. Long Range Planning, 37(4), 319-334.

[15] Lin, J. (2020, January). How do e-commerce capabilities influence agricultural firm performance gains? Theory and empirical evidence. In Proceedings of the 53rd Hawaii International Conference on System Sciences.

[16] Mann, S., \& Holdsworth, L. (2003). The psychological impact of teleworking: stress, emotions and health. New Technology, Work and Employment, 18(3), 196-21.

[17] Martin, B. H., \& MacDonnell, R. (2012). Is telework effective for organizations?. Management Research Review.

[18] Nandhakumar, J., \& Baskerville, R. (2006). Durability of online teamworking: patterns of trust. Information Technology \& People, 19(4), 371-389.

[19] Panteli, N., \& Duncan, E. (2004). Trust and temporary virtual teams: alternative explanations and dramaturgical relationships. Information Technology \& People.

[20] Petrie, D. E. (2004). Understanding the impact of technological discontinuities on information systems management: the case of business-to-business electronic commerce (Doctoral dissertation, The Claremont Graduate University).

[21] Rothaermel, F. T. (2016). Competitive advantage in technology intensive industries. In Technological innovation: Generating economic results. Emerald Group Publishing Limited.

[22] Silva-C, A. (2019). The attitude of managers toward telework, why is it so difficult to adopt it in organizations?. Technology in Society, 59, 101133.

[23] Tushman, M. L., \& Anderson, P. (1986). Technological discontinuities and organizational environments. Administrative science quarterly, 439465.

[24] Zhang, M. J., \& Lado, A. A. (2001). Information systems and competitive advantage: a competencybased view. Technovation, 21(3), 147-156. 